\title{
Numerical Analysis of the Aerodynamic Characteristics of NACA-4312 Airfoil
}

\author{
Md Rhyhanul Islam Pranto*, Mohammad Ilias Inam \\ Department of Mechanical Engineering, Khulna University of Engineering \& Technology, Khulna-9203, BANGLADESH
}

Received: May 12, 2020, Revised: June 17, 2020, Accepted: June 18, 2020, Available Online: June 24, 2020

\begin{abstract}
The aim of the work is to investigate the aerodynamic characteristics such as lift coefficient, drag coefficient, pressure distribution over a surface of an airfoil of NACA-4312. A commercial software ANSYS Fluent was used for these numerical simulations to calculate the aerodynamic characteristics of 2-D NACA-4312 airfoil at different angles of attack $(\alpha)$ at fixed Reynolds number $(R e)$, equal to $5 \times 10^{5}$. These simulations were solved using two different turbulence models, one was the Standard $k-\varepsilon$ model with enhanced wall treatment and other was the SST $k-\omega$ model. Numerical results demonstrate that both models can produce similar results with little deviations. It was observed that both lift and drag coefficient increase at higher angles of attack, however lift coefficient starts to reduce at $\alpha=13^{\circ}$ which is known as stalling condition. Numerical results also show that flow separations start at rare edge when the angle of attack is higher than $13^{\circ}$ due to the reduction of lift coefficient.
\end{abstract}

Keywords: Airfoil; CFD; RANS; Lift Coefficient; Drag Coefficient.

This work is licensed under a Creative Commons Attribution-NonCommercial 4.0 International

\section{Introduction}

Aerodynamics is defined as a study of the resulting effects of relative motion between air molecules and body surfaces [1]. Understanding the air flow behavior around a moving body is very important because we can control the air flow to our advantage in the case of aircraft, wind turbine, drone, and many more. Aerodynamics focuses on studying this phenomenon by applying basic physics laws such as Newton's laws and the Navier-Stokes equation. To study the aerodynamic profile of a particular object, it is necessary to define the object's shape, where the airfoil or the wing cross-section is used in the case of an airplane. The cross-section of the typical aircraft wing is an airfoil and is largely responsible for producing the forces that sustain the aircraft in flight [2]. Airfoil is known as the cross-sectional shape of a wing, blade (of a propeller, rotor, or turbine) which is placed in an airstream in order to generate useful aerodynamic forces. An airfoil's aerodynamic profile can be examined in two ways, one experimentally and other computationally. The aerodynamic characteristics of an airfoil are obtained in the experimental method by placing the airfoil (or wing model) in a controlled wind tunnel and recording the velocity and pressure distribution around the airfoil. The same experiment can be done in the computational approach using a CFD code. Both approaches are usually complementary to each other and can help us design a specific purpose for the airfoil [3].

In the early 1800 s, it was discovered that a curved surface produces more lift than a similar size flat plate by Sir George Cayley [4]. The most effective way to do this is to use an airfoil. In the Langley two-dimensional low turbulence tunnels tests a considerable amount of airfoil data has been accumulated by National Advisory Committee for Aeronautics (NACA) in the United States. The development of NACA airfoils which are now in common use was started in 1929 with a systematic investigation of a family of airfoils in the Langley variabledensity tunnel [5]. Airfoils of this family are designated by numbers having four digits. Experiments were performed to test different airfoils and to determine the lift and drag coefficient for different airfoils and to report their results. Jacobs et al. (1933) performed a wide variety of experiments comparing the geometric airfoil parameters and angle of attack and wind speed for different NACA airfoils and published the results of the lift and drag coefficient for 78 different NACA airfoils [6]. Since these experiments were carried out in a wind tunnel with a relatively high free-stream turbulence intensity of about $2 \%$, the results are expected to vary from low free-stream turbulence conditions. NACA airfoils have been used extensively as aerodynamic test models. NACA-0012 and NACA-4412 profiles are arguably the most studied airfoils. Ravi et al. [7] predicted numerically a transition model of an incompressible laminar to turbulent flow over NACA-4412 airfoil at Reynolds number of $3 \times 10^{6}$. Eleni et al. [8] also carried out studies on the variation of lift and drag coefficients from flow around NACA-0012 airfoil at Reynolds number of $3 \times 10^{6}$ for different turbulence models. In addition to the results available for a NACA-0012 and NACA-4412 airfoil profile, a substantial experimental or numerical database is not available for NACA4312 airfoil. This study is motivated by the need to complement the presently limited body of knowledge for NACA-4312 airfoil. Thus, the present investigation is focused on examining the effect of the Reynolds number and the angle of attack on the performance characteristics of a NACA-4312 airfoil and relating the performance characteristics.

In this study, CFD approach is used to determine the aerodynamic profiles of the NACA-4312 airfoil. The CFD packages contain three main elements which are Pre-processor, Solver and Post-processor [9]. Recently CFD has been the method of choice in the aerospace, automotive and many industrial components. CFD is vastly used in the field of aerodynamics because it is cheaper than experimental process and also gives more accurate results. The transition from the laminar to the turbulent flow phase plays a very important role in simulating the flow over an airfoil. The fluid flow over the airfoil exerts a pressure force perpendicular to the upper and lower 
surfaces along with shear force which is parallel to the surfaces. The resultant of these two forces is the area of interest. The normal component of the force is known as lift force and the force which is acting in the flow direction is known as drag [10].

It was observed from the previous literature review that there is a lack of understanding of the characteristics of NACA-4312 airfoil. In this paper a series of numerical simulations were carried out to analyze the characteristics of NACA-4312 airfoil with cord length of $1000 \mathrm{~mm}$ and Reynolds number of $5 \times 10^{5}$. ANSYS Fluent was used to solve the steady-state RANS (Reynolds Average Navier-Stokes) equation with two different turbulence models.

\section{Methodology}

\subsection{Airfoil}

In this paper, NACA-4312 airfoil was selected for the simulations, the profile is shown in Fig. 1. The NACA-4312 airfoil means that it has a maximum camber equal to $4 \%$ of the cord which is located at $30 \%$ of the cord from the leading edge with a maximum thickness of $12 \%$ of the chord. In this paper, cord length was considered $1000 \mathrm{~mm}$. Airfoil was created by ANSYS design modeler by importing coordinate file of NACA-4312.

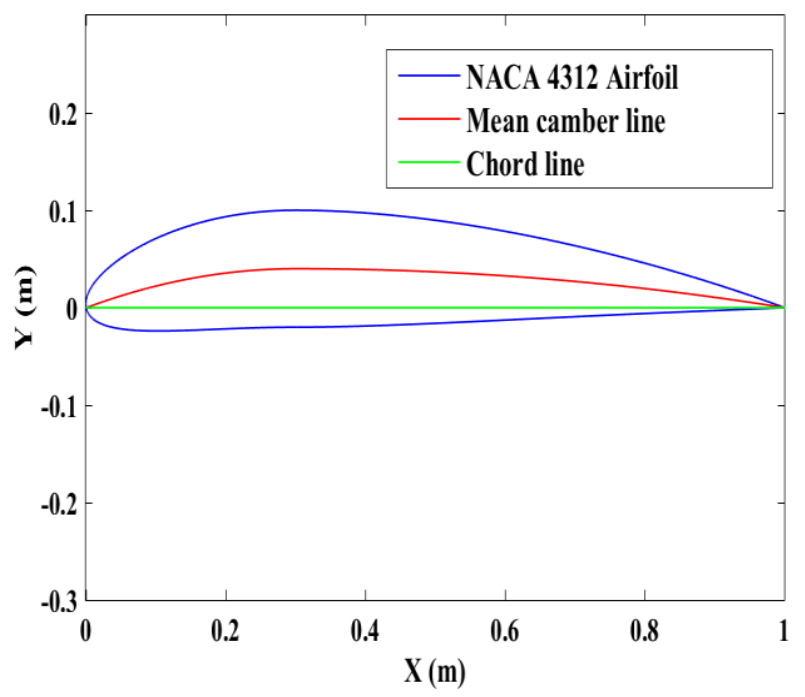

Fig. 1 Geometry of NACA-4312 airfoil.

\subsection{Computational Method}

These simulations were conducted by ANSYS Fluent. The problem was solved in steady-state with two turbulence models. These simulations were conducted at fixed Reynolds number $(R e)$, equal to $5 \times 10^{5}$, to show the effect at the transition region [11]. Air was assumed as working medium with a constant density $(\rho)$ and viscosity $(\mu)$, whereas $\rho=$ $1.225 \mathrm{~kg} / \mathrm{m}^{3}$, and $\mu=1.7894 \times 10^{-5} \mathrm{~kg} / \mathrm{ms}$ [12]. The steadystate RANS (Reynolds Average Navier-Stokes) equation was solved using the Green-Gauss cell based gradient option and pressure based solver was selected as the flow is incompressible. The RANS equations are timeaveraged equations of motion for fluid flow, as bellows:

$$
\frac{\partial}{\partial x_{i}}\left(\rho u_{i}\right)=0
$$

$$
\begin{aligned}
& \frac{\partial}{\partial x_{j}}\left(\rho u_{i} u_{j}\right)= \\
& \frac{\partial p}{\partial x_{i}}+\frac{\partial}{\partial x_{j}}\left[\mu\left(\frac{\partial u_{i}}{\partial x_{j}}+\frac{\partial u_{j}}{\partial x_{i}}-\frac{2}{3} \delta_{i j} \frac{\partial u_{l}}{\partial x_{l}}\right)\right]+\frac{\partial}{\partial x_{j}}\left(-\rho \overline{u_{i}^{\prime} u_{j}^{\prime}}\right)
\end{aligned}
$$

where $\rho$ indicates density, $u$ indicates velocity and $\mu$ indicates dynamic viscosity of the fluid. The left side of this equation describes the fluid element's mean momentum change to the flow instability and convection by the mean flow. This adjustment is regulated by the mean body force, the isotropic stress due to the mean pressure field, the viscous stresses, and visible stress due to the fluctuating velocity field, commonly referred to as the Reynolds stress $\left(-\rho \overline{u_{\imath}^{\prime} u_{\jmath}^{\prime}}\right)$. This nonlinear stress term requires additional modeling to solve the RANS equations and has resulted in many different turbulence models [13].

In this paper, the Standard $k-\varepsilon$ model and the SST $k-$ $\omega$ model are used to predict the effects of turbulence in flow over the airfoil.

\subsubsection{Standard k- $\varepsilon$ Model}

$\mathrm{K}$-epsilon $(k-\varepsilon)$ turbulence model is the most common model used in Computational Fluid Dynamics (CFD) for simulating flow characteristics for turbulent flow conditions. It is a two-equation model, which provides a general definition of turbulence via solving two transport equations (PDEs) one for the turbulence kinetic energy $(\mathrm{k})$ and the other is its dissipation rate $(\varepsilon)$ [13]. The turbulent kinetic energy $\mathrm{k}$ and dissipation $\varepsilon$ are obtained from the following transport equations (3) and (4):

$$
\begin{aligned}
& \frac{\partial}{\partial x_{i}}\left(\rho k u_{i}\right)= \\
& \frac{\partial}{\partial x_{j}}\left[\left(\mu+\frac{\mu_{t}}{\sigma_{k}}\right) \frac{\partial k}{\partial x_{j}}\right]+G_{k}+G_{b}-\rho \varepsilon-Y_{M}+S_{k} \\
& \frac{\partial}{\partial x_{i}}\left(\rho \varepsilon u_{i}\right)=\frac{\partial}{\partial x_{j}}\left[\left(\mu+\frac{\mu_{t}}{\sigma_{\varepsilon}}\right) \frac{\partial \varepsilon}{\partial x_{j}}\right] \\
& +C_{1 \varepsilon} \frac{\varepsilon}{k}\left(G_{k}+C_{3 \varepsilon} G_{b}\right)-C_{2 \varepsilon} \rho \frac{\varepsilon^{2}}{k}+S_{\varepsilon}
\end{aligned}
$$

In these equations,

$\mathrm{G}_{\mathrm{k}}=$ Generation of turbulence kinetic energy due to mean velocity gradient

$\mathrm{Y}_{\mathrm{M}}=$ Fluctuation in compressible turbulence to overall dissipation rate

$C_{1 \varepsilon}, C_{2 \varepsilon}, C_{3 \varepsilon}=$ Constants

$\sigma_{k}, \sigma_{\varepsilon}=$ Turbulent Prandtl number for $\mathrm{k}$ and $\varepsilon$

$S_{k}, S_{\varepsilon}=$ User defined source terms

Here turbulent viscosity $\mu_{t}=\rho C_{\mu} \frac{k^{2}}{\varepsilon}$; where is $C_{\mu}$ a constant.

\subsubsection{SST k- $\omega$ Model}

In CFD analysis SST (Shear Stress Transport) $k-\omega$ is a widely used and two-equation eddy-viscosity turbulence model. It provides a general solution for the turbulence kinetic energy $(\mathrm{k})$ and specific dissipation rate of eddy viscosity $(\omega)$. This model gives more accurate results in numerical flow 
analysis of airfoil than the standard $k-\omega$ turbulence model as it includes transportation of turbulence sheer stress. The proper transport behavior can be obtained to the formulation of eddyviscosity [14].The turbulent kinetic energy $\mathrm{k}$ and dissipation rate of eddy viscosity $\omega$ are obtained from the following transport equations (5) and (6):

$$
\begin{aligned}
& \frac{\partial}{\partial x_{i}}\left(\rho k u_{i}\right)=\frac{\partial}{\partial x_{j}}\left[\left(\mu+\sigma_{k} \mu_{t}\right) \frac{\partial k}{\partial x_{j}}\right]+G_{k}-Y_{k}+S_{k} \\
& \frac{\partial}{\partial x_{i}}\left(\rho \omega u_{i}\right)=\frac{\partial}{\partial x_{j}}\left[\left(\mu+\sigma_{\omega} \mu_{t}\right) \frac{\partial \omega}{\partial x_{j}}\right]+G_{\omega}-Y_{\omega}+S_{\omega}
\end{aligned}
$$

In these equations,

$\mathrm{G}_{\mathrm{k}}=$ Generation of turbulence kinetic energy due to mean velocity gradient

$G_{\omega}=$ Generation of $\omega$

$Y_{k}, Y_{\omega}=$ The dissipation of $\mathrm{k}$ and $\omega$

$\sigma_{k}, \sigma_{\omega}=$ Turbulent Prandtl number for $\mathrm{k}$ and $\omega$

$S_{k}, S_{\omega}=$ User defined source terms

Here turbulent viscosity $\mu_{t}=\frac{\rho k}{\omega} \frac{1}{\max \left[\frac{1}{\alpha^{*},} \frac{S F_{2}}{\alpha_{1} \omega}\right.}$; where $\mathrm{S}$ is the strain rate magnitude, $\mathrm{F}_{2}$ is a constant, and $\propto=$ Angle of attack.

\subsection{Boundary Condition with domain}

For these simulations, a computation domain was created around the NACA-4312 airfoil, shown in Fig. 1. Chord length of NACA-4312 airfoil was assumed to be equal to $1000 \mathrm{~mm}$. To minimize the boundary effect, the domain was extended 12.5C (C indicated chord length) along the upstream and 20C along downstream from the trailing edge, like Fig. 2 [15]. In the domain, airfoil surface was assumed no-slip boundary condition, BAFED was assumed constant velocity inlet and pressure outlet at BCD. For this fixed $R e$, the inlet velocity $u$, was assumed to $7.5 \mathrm{~m} / \mathrm{s}$. In these simulation angle of attack $(\alpha)$ was changed by changing flow direction instead of rotating airfoil which produced the same effect on the airfoil. The velocity components along $x$ and $y$ direction were calculated by $u \cos \alpha$ and $u \sin \alpha$, respectively, for different angle of attack $(\alpha)$.

\subsection{Mesh generation and Wall Treatment}

The C-type structural mesh was created for better convergence and control of the wall function, shown in Fig. 3 and Fig. 4. Fig. 3 depicts the structural meshing with quadrilateral elements which was done by ANSYS Meshing whereas Fig. 4 illustrates the meshing quality around the airfoil profile.

The application of wall functions near the wall region may significantly reduce the processing and storage requirements while producing an acceptable degree of solution. To ensure sufficient boundary layer modeling the inflation was set to 1.15.

The effectiveness of $k-\varepsilon$ model is beyond $y^{+}=30$. The non-dimensional wall parameter is defined as:

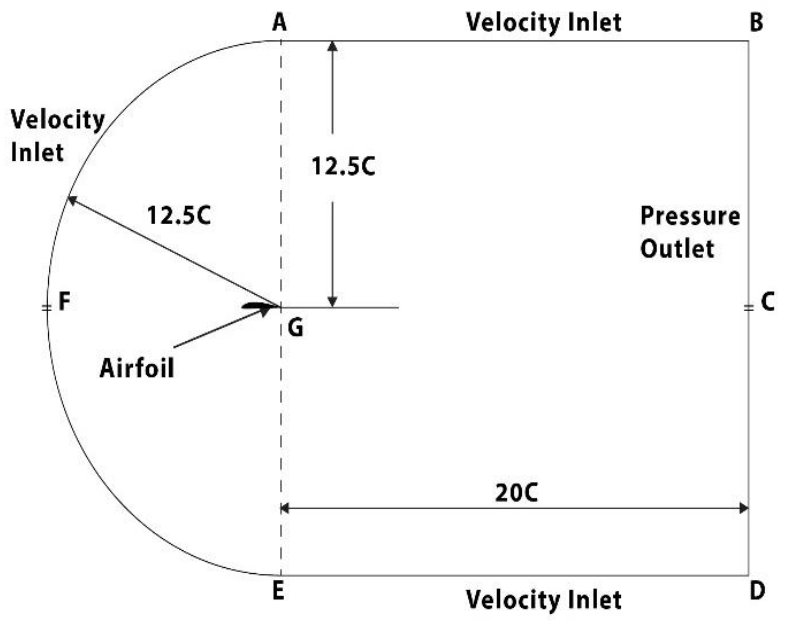

Fig. 2 Computation domain with boundary conditions.

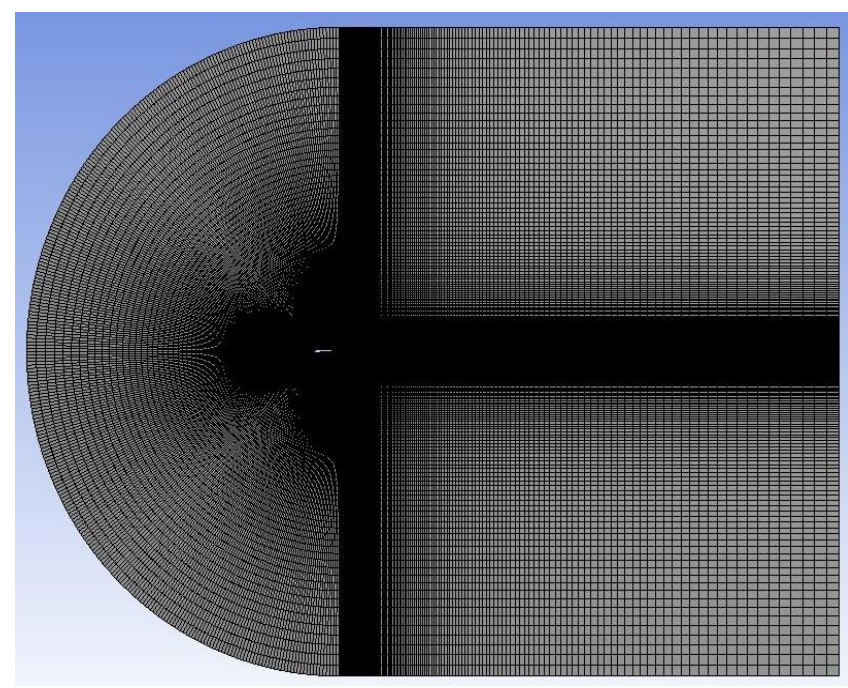

Fig. 3 Mesh among the whole domain.

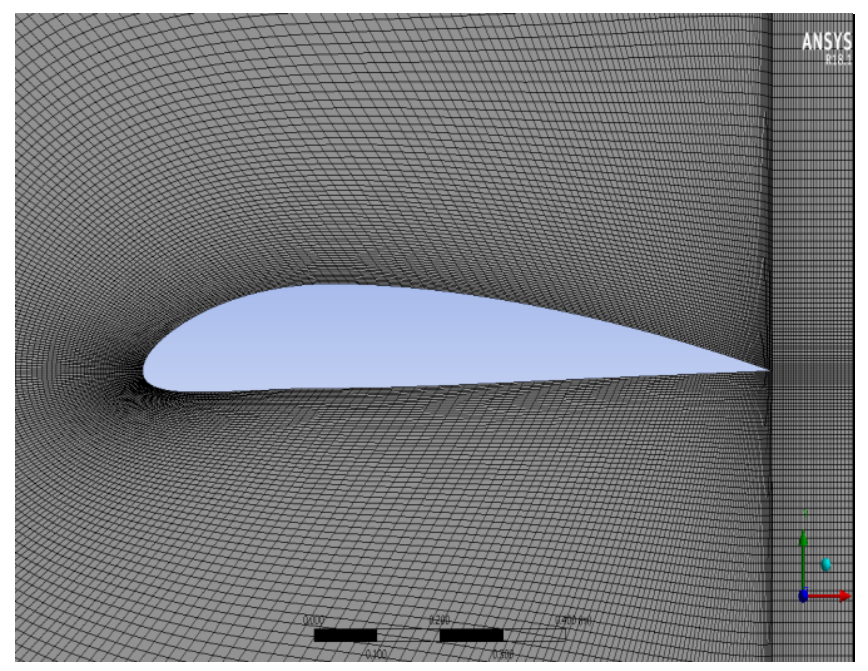

Fig. 4 Mesh around the NACA-4312 airfoil. 
$y^{+}=y \times \frac{\sqrt{(\tau \omega / \rho)}}{\mu}$

Here $y$ is the distance from the wall to the centroid of the first fluid cell. It was observed for all simulations that the value of $y^{+}$was higher than 30 and lower than 60 .

\subsection{Mesh Independence Test}

A series of simulations were conducted for mesh independency tests. A different number of meshes were created by diving circular and rectangular sections with different numbers. Fig. 5 depicts the variation of lift coefficient for different numbers of elements of the mesh for an angle of attack $5^{\circ}$. It is observed from figure that meshes with higher than 105000 number of elements can produce accurate results with minimum deviation. As a result mesh with element number 105000 were selected for further simulation.

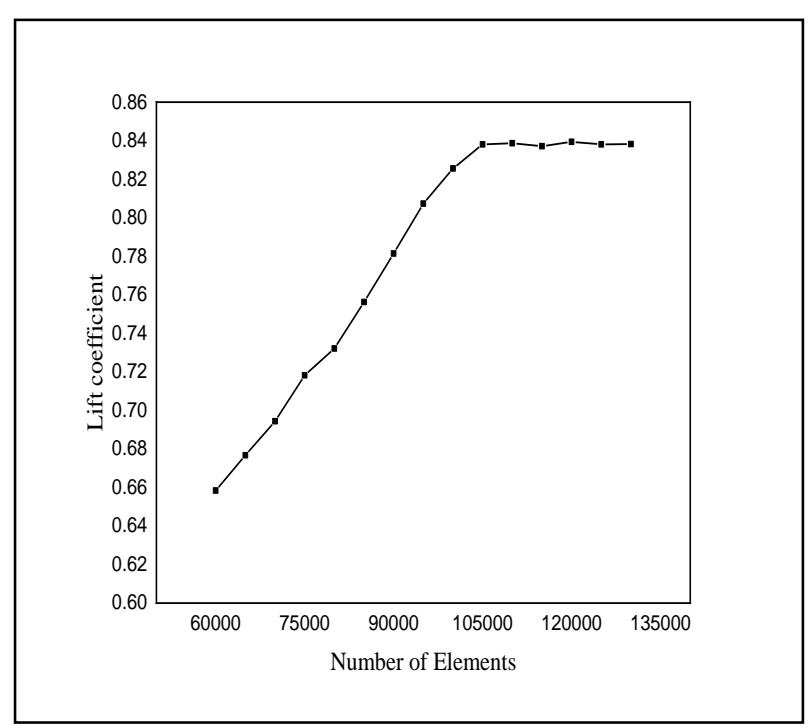

Fig. 5 Variation of coefficient of lift with no. of element.

\section{Results and Discussion}

Fig. 6 depicts the variation of lift coefficient for different angles of attack for two different turbulence models. It is observed that the lift coefficients are almost equal for both models up to $\alpha=6^{0}$, however, the SST $k-\omega$ model predicts higher lift coefficient compared to the standard $k-\varepsilon$ model when $\alpha>6^{0}$. Fig. 6 also shows that the lift coefficient increases with angle of attack, for both models, up to $12^{\circ}$. Later on, it decreases with $\alpha$ due to the flow separation at the trailing edge, which indicated stalling occurs at the range of $12^{0} \sim 13^{0}$. From $0^{\circ}$ angle of attack to $12^{\circ}$ angle of attack the lift curve is almost linear.

Fig. 7 shows the effect of the angle of attack $(\alpha)$ on the drag coefficient for two different turbulence method. It is observed that the drag coefficient estimates higher values with the standard $k-\varepsilon$ model compared to the SST $k-\omega$ because of the transportation of the shear viscosity. For both models, the drag coefficient increase with $\alpha$, however, the increment rate is higher at higher $\alpha$ due to the flow separation at trailing edge. The drag coefficient still increases after the stalling angle however lift coefficient decreases.

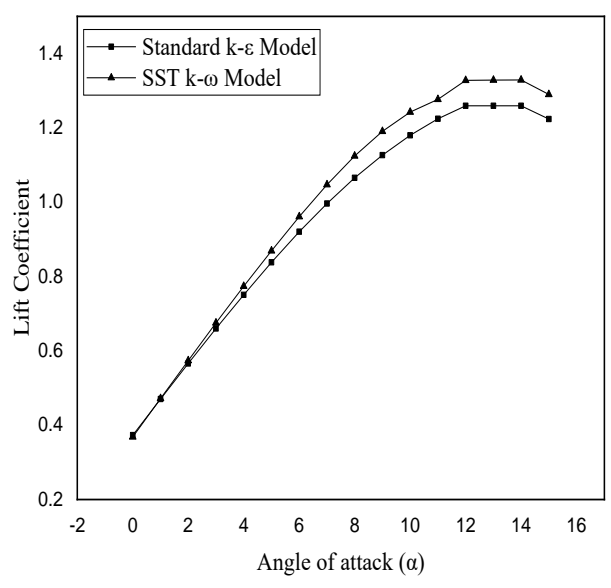

Fig. 6 Lift coefficient vs angle of attack.

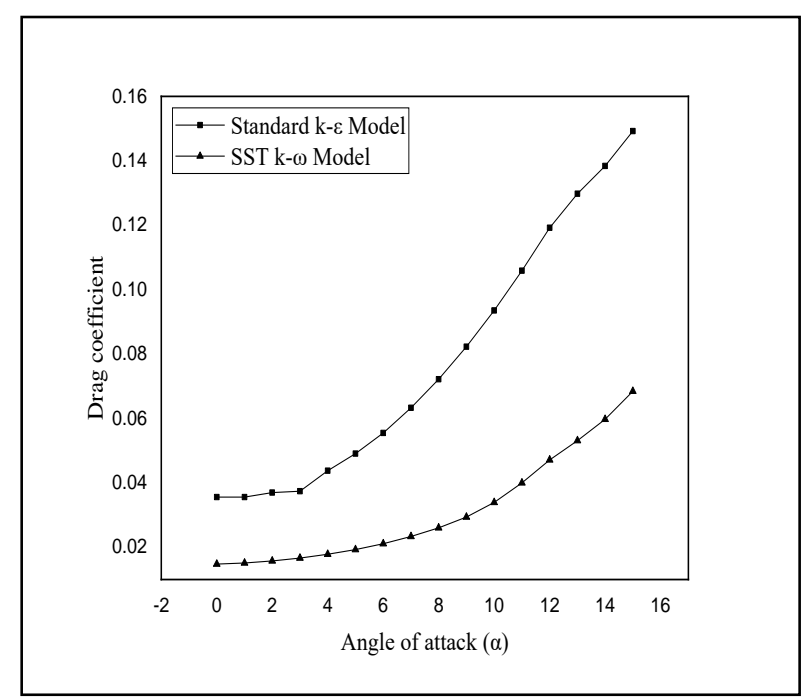

Fig. 7 Drag coefficient vs angle of attack.

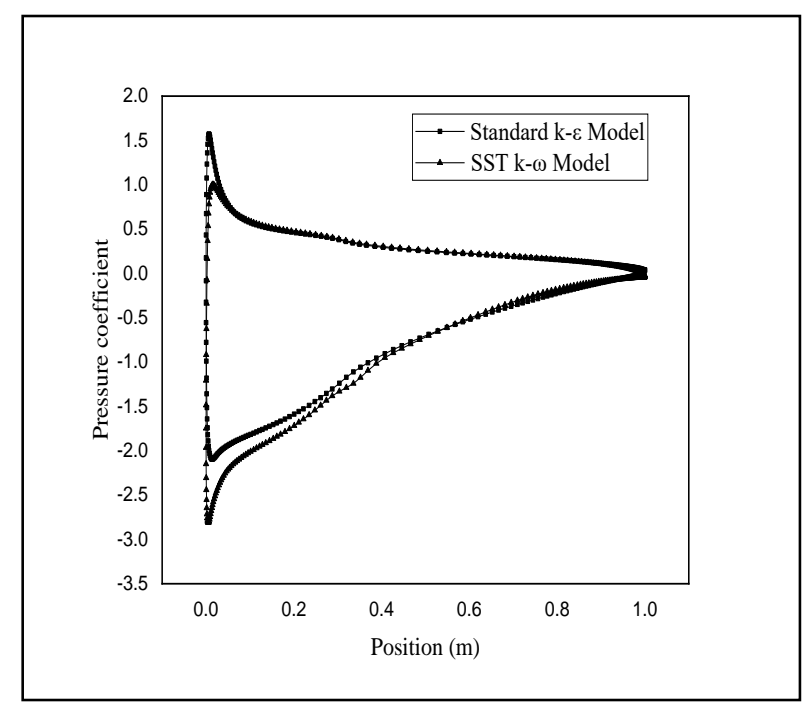

Fig. 8 Pressure coefficient on the airfoil surface at $10^{\circ}$ angle of attack. 


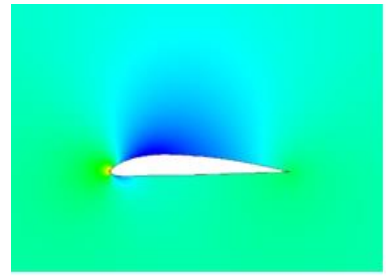

(a) $\theta=0^{\circ}$

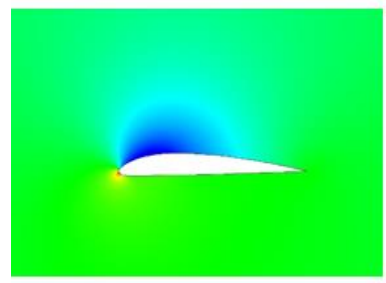

(e) $\theta=5^{\circ}$

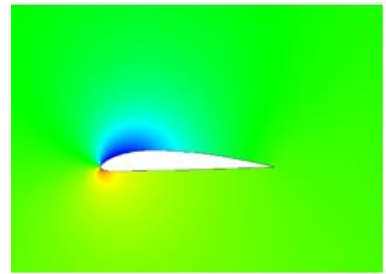

(i) $\theta=9^{\circ}$

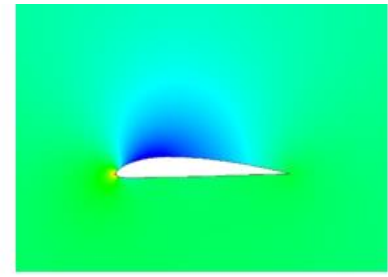

(b) $\theta=2^{\circ}$

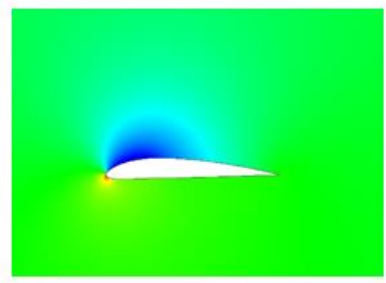

(f) $\theta=6^{\circ}$

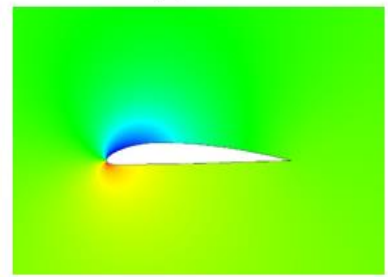

(j) $\theta=10^{\circ}$

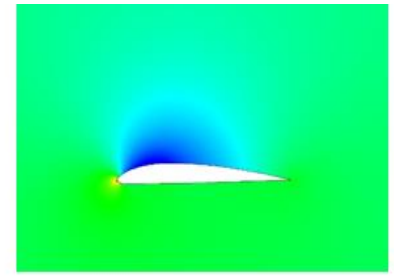

(c) $\theta=3^{\circ}$

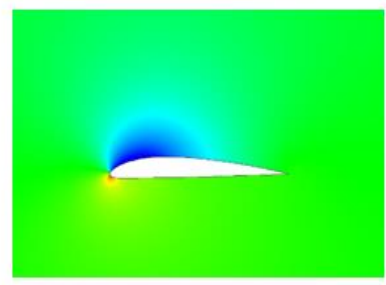

(g) $\theta=7^{\circ}$

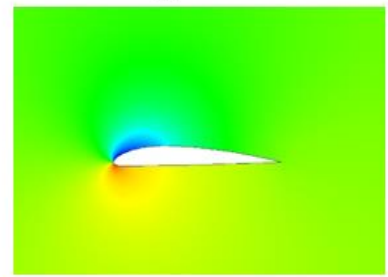

(k) $\theta=11^{\circ}$

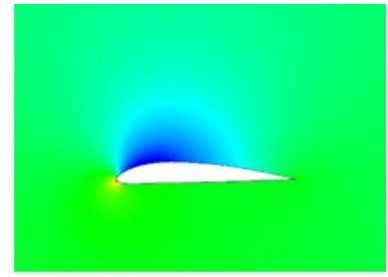

(d) $\theta=4^{\circ}$

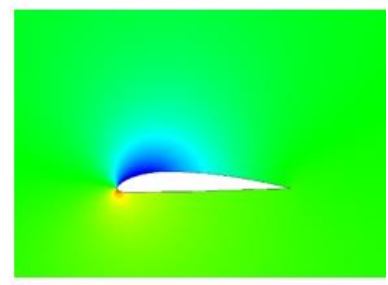

(h) $\theta=8^{\circ}$

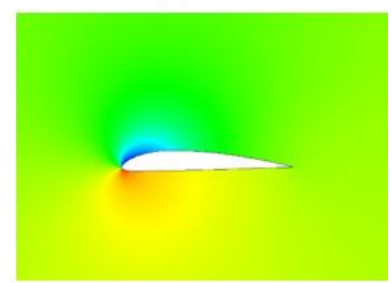

(l) $\theta=12^{\circ}$

contour-1
Pressure coefficient

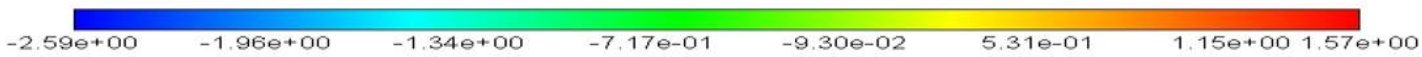

Fig. 9 Contours of pressure with the Standard k- $\varepsilon$ model for different angles of attack of NACA-4312 airfoil.

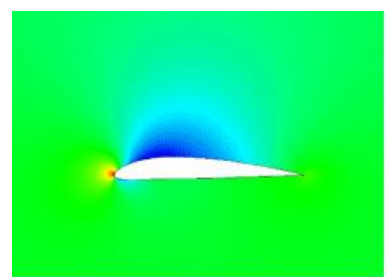

(a) $\theta=0^{\circ}$

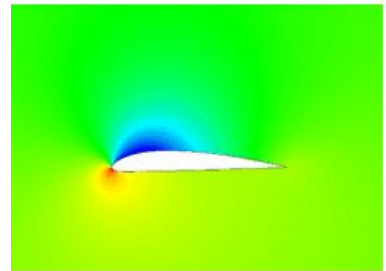

(e) $\theta=5^{\circ}$

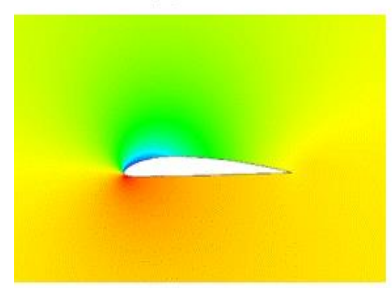

(i) $\theta=9^{\circ}$

contour-1 Pressure Coefficient

-2.81800

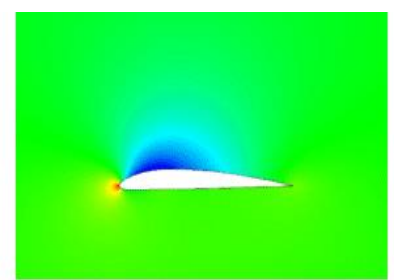

(b) $\theta=2^{\circ}$

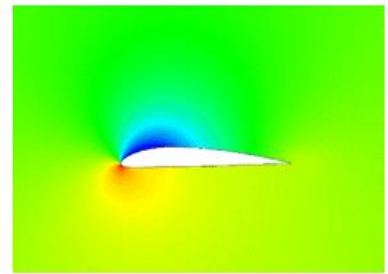

$(f) \theta=6^{\circ}$

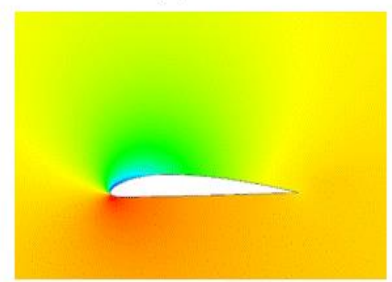

(j) $\theta=10^{\circ}$

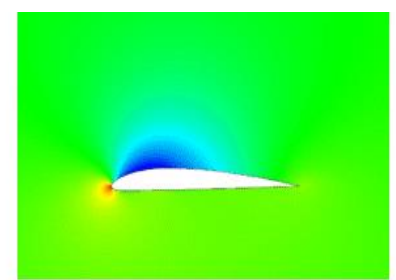

(c) $\theta=3^{\circ}$

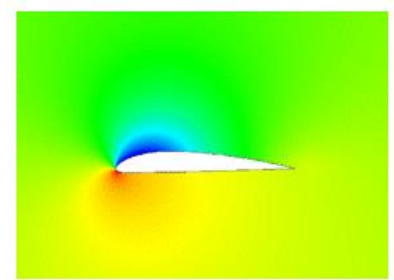

(g) $\theta=7^{\circ}$

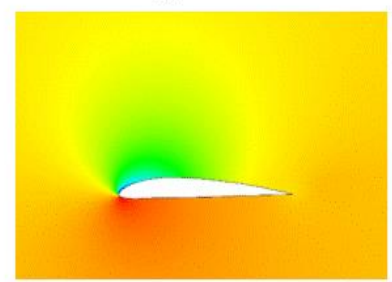

(k) $\theta=11^{\circ}$

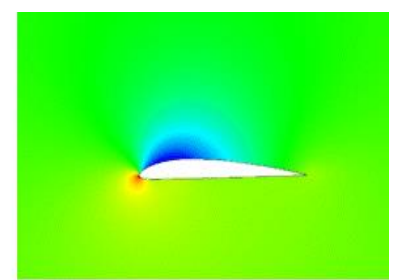

(d) $\theta=4^{\circ}$

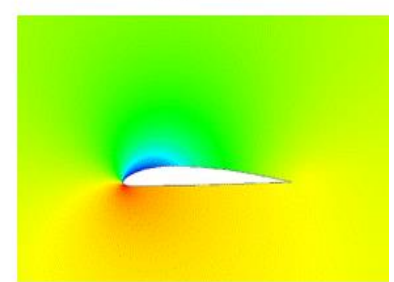

(h) $\theta=8^{\circ}$

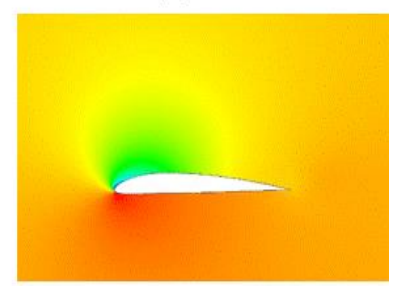

(l) $\theta=12^{\circ}$

Fig. 10 Contours of pressure with the SST k- $\omega$ model for different angles of attack of NACA-4312 airfoil. 


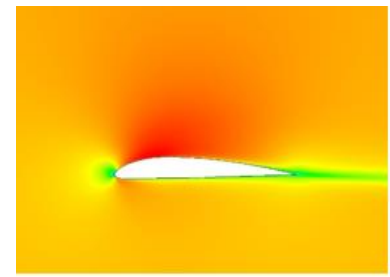

(a) $\theta=0^{\circ}$

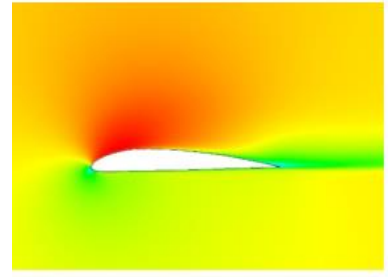

(e) $\theta=5^{\circ}$

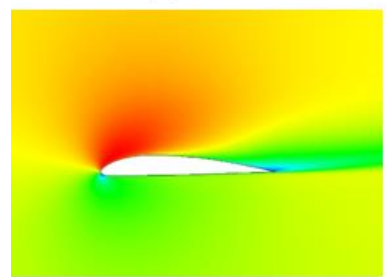

(i) $\theta=9^{\circ}$

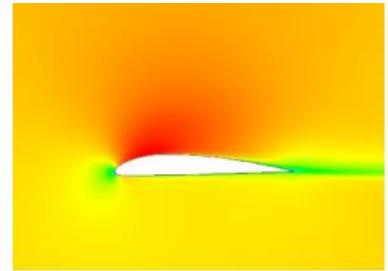

(b) $\theta=2^{\circ}$

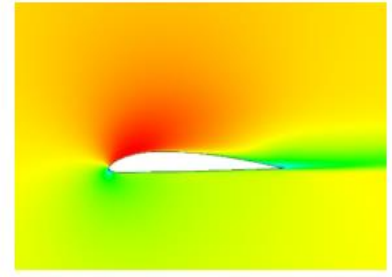

(f) $\theta=6^{\circ}$

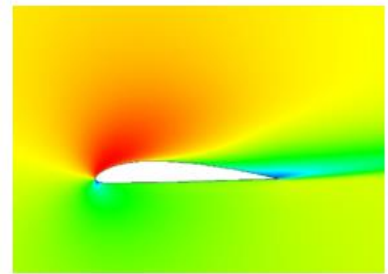

(j) $\theta=10^{\circ}$

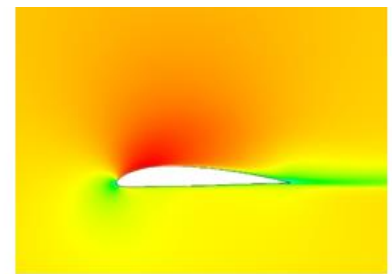

(c) $\theta=3^{\circ}$

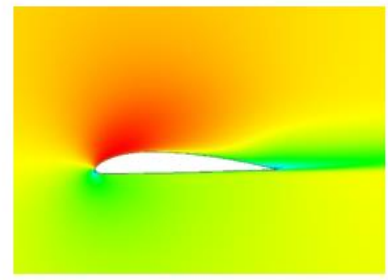

(g) $\theta=7^{\circ}$

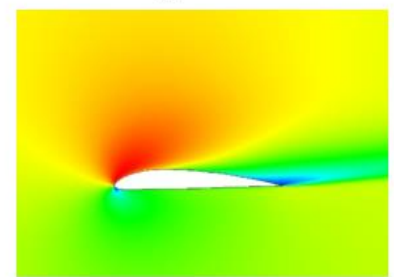

(k) $\theta=11^{\circ}$

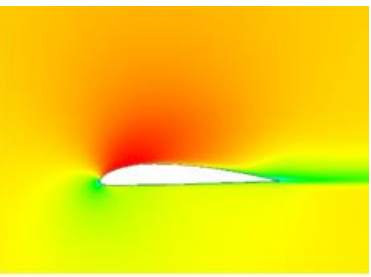

(d) $\theta=4^{\circ}$

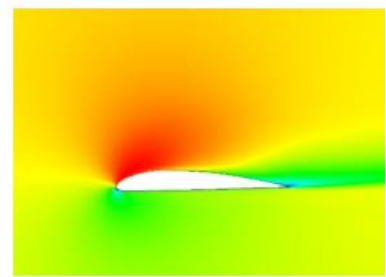

(h) $\theta=8^{\circ}$

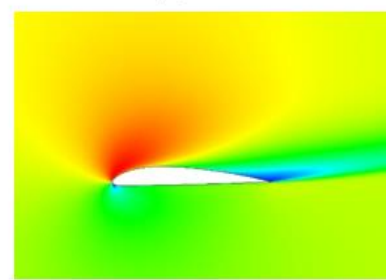

(l) $\theta=12^{\circ}$

contour-1
Velocity Magnitude

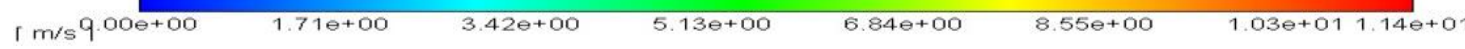

Fig. 11 Velocity contours with the Standard k- $\varepsilon$ model

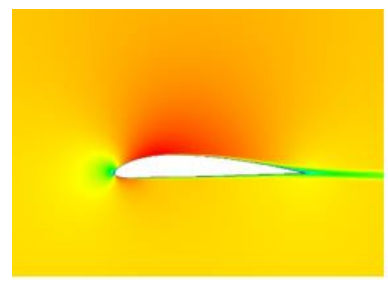

(a) $\theta=0^{\circ}$

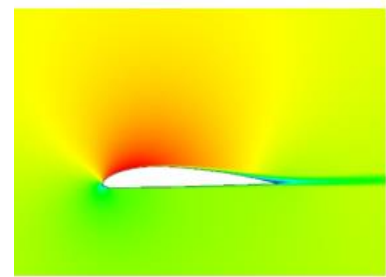

(e) $\theta=5^{\circ}$

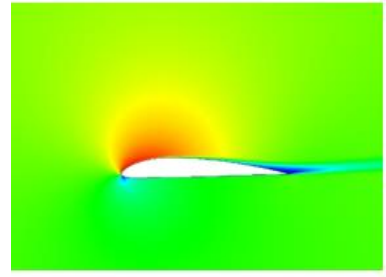

(i) $\theta=9^{\circ}$ contour-1
velocity Magnitude

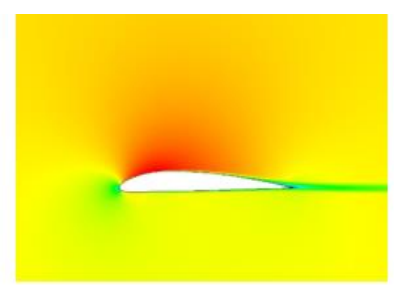

(b) $\theta=2^{\circ}$

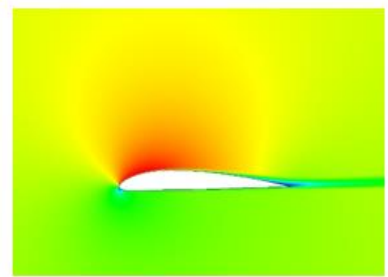

(f) $\theta=6^{\circ}$

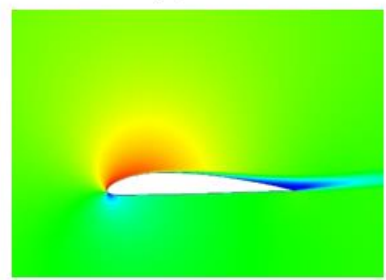

(j) $\theta=10^{\circ}$

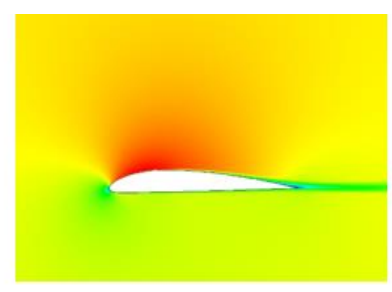

(c) $\theta=3^{\circ}$

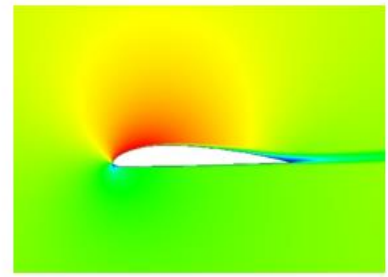

(g) $\theta=7^{\circ}$

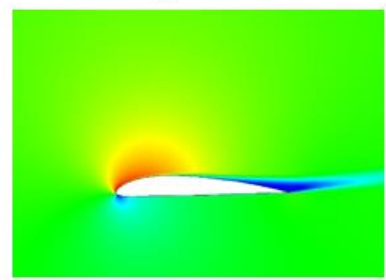

(k) $\theta=11^{\circ}$

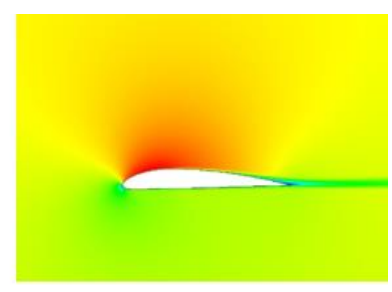

(d) $\theta=4^{\circ}$

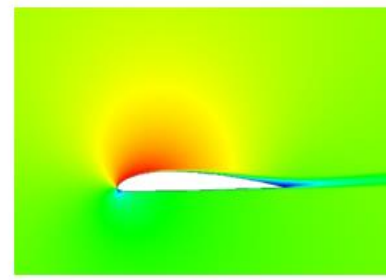

(h) $\theta=8^{\circ}$

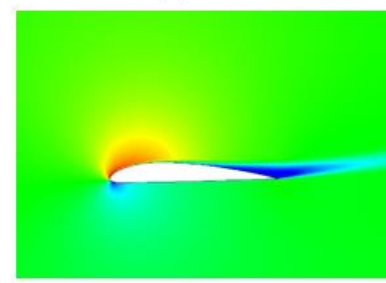

(l) $\theta=12^{\circ}$

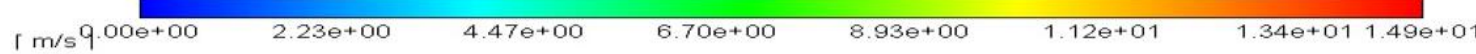

Fig. 12 Velocity contours with the SST k- $\omega$ model 
Fig. 8 demonstrates pressure distribution on the airfoil surface for both models at an angle of attack $\alpha=10^{\circ}$. The upper and lower portion of the graph represent pressure coefficient distribution on the lower surface and upper surface of the airfoil, respectively. It is observed that the upper surface have lower pressure and lower surface have higher pressure which produces lift. Maximum lower and higher pressure observed near to the leading of the lower and upper surface, respectively, which starts to reduce along the trailing edge.

\subsection{Pressure Contours}

Fig. 9 and Fig. 10 presents pressure contours for the Standard $k-\varepsilon$ turbulence model and the SST $k-\omega$ turbulence model, respectively, at different angle of attack. In these two figures, the effect of angles of attack is shown by changing the flow directions instead of rotating airfoil, which gives the same effect on airfoil. Similar pressure distribution pattern is observed for the Standard $k-\varepsilon$ and SST $k-\omega$ turbulence model, present in Fig. 9 and Fig. 10, respectively at lower angle of attack, whereas slightly varies in magnitude at higher angle of attack though the pattern of pressure distribution is similar. It is clearly observed from both figures that the upper surface has lower pressure and the lower surface has higher pressure. It is also observed from both figures that negative pressure region on the upper surface which is situated almost around the whole surface which starts to decrease with the increase of angle of attack, however pressure on the lower surfaces increases with angle of attack. Though pressure difference between lower and upper surface increase until flow separates on the upper surface at a certain angle of attack. The lift coefficient increases in both turbulence model with the increase of angle of attack and it reaches the stalling position near $13^{\circ}$ angle of attack. Between the two turbulence models, it is observed that the pressure difference in the SST $k-\omega$ model is slightly higher than the Standard $k-\varepsilon$ model which is clearly observed in Fig. 8 also.

\subsection{Velocity Contours}

Fig. 11 and Fig. 12 depict velocity contours for the Standard $k-\varepsilon$ turbulence model and SST $k-\omega$ turbulence model, respectively, at different angles of attack. In these two figures, the effect of angle of attack is shown by changing the flow directions instead of rotating airfoil, which gives the same effect on airfoil. Similar velocity distribution pattern is observed for the Standard $k-\varepsilon$ and SST $k-\omega$ turbulence model, present in Fig. 11 and Fig. 12, respectively at lower angle of attack, whereas slightly varies in magnitude at higher angle of attack though patter of velocity distribution is similar. It is clearly observed from both figures that velocity above the upper is higher than the velocity below the lower surface. This happens due to the Bournollie's principle. It is also observed from both figures that at lower angle of attack flow is attached to upper surface and lower surface as a result there is no zone of zero velocity which indicates flow separation happens. When the angle attack reaches to the value of $10^{\circ}$ flow starts to separate, see Fig. 11, at the trailing edges which starts to move towards the leading edges at higher angles of attack. A similar pattern is also observed for the SST $k-\omega$ model, see Fig. 12, however, flow separation is identified comparative lower angle of at $8^{0}$ compare to the Standard $k-\varepsilon$ model. Due to this flow separation, lift coefficient starts to decrease after a certain angle of attack.

\section{Conclusion}

In this paper aerodynamic characteristics of NACA-4312 airfoil have been studied using ANSYS Fluent by the Standard $\boldsymbol{k}-\boldsymbol{\varepsilon}$ model and SST $\boldsymbol{k}-\boldsymbol{\omega}$ model turbulence model. At lower angles of attack both methods produce similar results however at higher angles of attack results vary in magnitude slightly though nature is similar. It is observed that the lift and drag coefficient increase with angle of attack though the lift coefficient shows a decreasing trend beyond the angle of attack $13^{0}$.

\section{References}

[1] Fearn, R.L., 2008. Airfoil aerodynamics using panel methods. The Mathematica Journal, 10(4), p.15.

[2] Triet, N.M., Viet, N.N. and Thang, P.M., 2015. Aerodynamic analysis of aircraft wing. VNU Journal of Science: Mathematics-Physics, 31(2).

[3] Sumaryada, T., Jaya, A.M. and Kartono, A., 2018. Simulating the Aerodynamics Profiles of NACA 4312 Airfoil in Various Incoming Airspeed and Gurney Flap Angle. Omega: Jurnal Fisika dan Pendidikan Fisika, 4(1), pp.1-1.

[4] Ackroyd, J.A.D., 2011. Sir George Cayley: the invention of the aeroplane near Scarborough at the time of Trafalgar. Journal of Aeronautical History Paper No, p.6.

[5] Oppermann, R.H., 1938. National advisory committee for aeronautics: Report No. 624, Two-Dimensional Subsonic Compressible Flow Past Elliptic Cylinders, by Carl Kaplan. 8 pages, illustrations, 23× $29 \mathrm{cms}$. Washington, Government Printing Office, 1938. Price 10 cents.

[6] Jacobs, E.N., Ward, K.E. and Pinkerton, R.M., 1933. The Characteristics of 78 related airfoil section from tests in the Variable-Density Wind Tunnel(No. 460). US Government Printing Office.

[7] Ravi, H.C., Madhukeshwara, N. and Kumarappa, S., 2013. Numerical investigation of flow transition for NACA-4412 airfoil using computational fluid dynamics. International Journal of Innovative Research in Science Engineering and Technology, 2(7), pp.2778-2785.

[8] Eleni, D.C., Athanasios, T.I. and Dionissios, M.P., 2012. Evaluation of the turbulence models for the simulation of the flow over a National Advisory Committee for Aeronautics (NACA) 0012 airfoil. Journal of Mechanical Engineering Research, 4(3), pp.100-111.

[9] Versteeg, H.K. and Malalasekera, W., 2007. An introduction to computational fluid dynamics: the finite volume method. Pearson education. 
[10] Cengel, Y.A. and Cimbala, J.M., 2012. Fluid Mechanics Fundamental and Aplication Third Edition.

[11] Incropera, F. P., DeWitt, D. P., Bergman, T. L. and Lavine, A. S., 2007. Fundamentals of Heat and Mass Transfer(6th edition).

[12] Marshall, J. and Plumb, R.A., 2008. Atmosphere, ocean and climate dynamics.

[13]Launder, B.E., 2015. First steps in modelling turbulence and its origins: a commentary on Reynolds (1895) 'On the dynamical theory of incompressible viscous fluids and the determination of the criterion'. Philosophical
Transactions of the Royal Society A: Mathematical, Physical and Engineering Sciences, 373(2039), p.20140231.

[14]Menter, F.R., 1994. Two-equation eddy-viscosity turbulence models for engineering applications. AIAA journal, 32(8), pp.1598-1605.

[15] Verhoff, A., Stookesberry, D. and Agrawal, S., 1992. Farfield computational boundary conditions for twodimensional external flow problems. AIAA journal, 30(11), pp.2585-2594. 